\title{
Case of Segmental Arterial Mediolysis
}

\author{
Maneka M Britto, ${ }^{1}$ Matthew Lukies, ${ }^{2}$ Charles Milne, ${ }^{3}$ Timothy Joseph, ${ }^{2}$ James C Lee
}

${ }^{1}$ Department of General Surgery, Alfred Health, Melbourne,

Victoria, Australia

2Department of Radiology,

Alfred Health, Melbourne,

Victoria, Australia

${ }^{3}$ Department of Vascular

Surgery, Alfred Hospital,

Melbourne, Victoria, Australia

Correspondence to

Dr Maneka M Britto,

manekabritto@gmail.com

Accepted 9 February 2018
Check for updates

To cite: Britto MM, Lukies M,

Milne C, et al. BMJ Case

Rep Published Online First:

[please include Day Month

Year]. doi:10.1136/bcr-2017-

223731

\section{SUMMARY}

Segmental arterial mediolysis (SAM) is a rare, noninflammatory, vascular condition that predominantly affects medium-sized to large-sized abdominal arteries and can present with haemorrhage into the abdominal cavity. We report the case of a patient with SAM of the coeliac, splenic, renal and gastroduodenal arteries in whom endovascular coil embolisation was successfully used to treat a bleeding gastroduodenal artery pseudoaneurysm.

\section{BACKGROUND}

It is important to consider vascular diseases such as occlusion, stenosis, dissection or rupture of an aneurysm in a patient who presents with a history of sudden onset abdominal pain. If the above clinical features are present concurrently, that would suggest a possible diagnosis of segmental arterial mediolysis (SAM). Therefore, we write this report to highlight that physicians and surgeons alike must consider SAM as a possible diagnosis in a patient who presents with abdominal pain and intra-abdominal haemorrhage as this could be life threatening, but can be treated endovascularly with good outcome and does not necessarily require open surgical intervention.

\section{CASE PRESENTATION}

A 69-year-old woman presented with acute onset abdominal pain without any systemic features. Her medical history included paroxysmal atrial fibrillation (for which she was on apixaban), hypertension, cardiac failure and a recent mitral valve repair. She had no known congenital abnormalities. On presentation, she was haemodynamically stable (initial haemoglobin $107 \mathrm{~g} / \mathrm{L}$ ) and afebrile. She was tender in the epigastrium and right lower quadrant without guarding. A CT scan showed a moderate-sized haemoperitoneum, associated with multiple aneurysms of the splanchnic arteries. The patient was fluid resuscitated and transferred to our tertiary hospital.

\section{INVESTIGATIONS}

On arrival, she remained stable, and a CT angiogram confirmed two pseudoaneurysms of the distal gastroduodenal artery (GDA) $-20 \mathrm{~mm}$ (with a surrounding haematoma, therefore the likely source of bleeding) and $9 \mathrm{~mm}$ in maximal dimension (figure 1A), a fusiform aneurysm and dissection flap of the celiac axis (figure 1B), a $10 \mathrm{~mm}$ fusiform aneurysm of the mid-splenic artery, a pseudoaneurysm of the distal splenic artery $(24 \mathrm{~mm})$ and multiple small fusiform aneurysms of both renal arteries.

\section{DIFFERENTIAL DIAGNOSIS}

Incorporated in the Discussion section.

\section{TREATMENT}

Her care was jointly provided by general and vascular surgeons, interventional radiologists and physicians. Two attempts were required to successfully occlude flow to the GDA. Given the dissection of the coeliac axis, catheter angiography via the superior mesenteric artery (SMA) was first attempted, but the GDA pseudoaneurysm was not accessible. It was then embolised percutaneously under ultrasound guidance using thrombin. Although no residual flow was demonstrated on ultrasound, repeat CT angiogram 2 days later showed persistent flow. Catheter angiography was then performed via the coeliac axis, and both GDA pseudoaneurysms were successfully coil embolised (figure 2A,B).

With the successful control of bleeding by the endovascular approach, a laparotomy was not performed for the purpose of obtaining a biopsy of the vessels. Therefore, we were unable to confirm the diagnosis histologically.

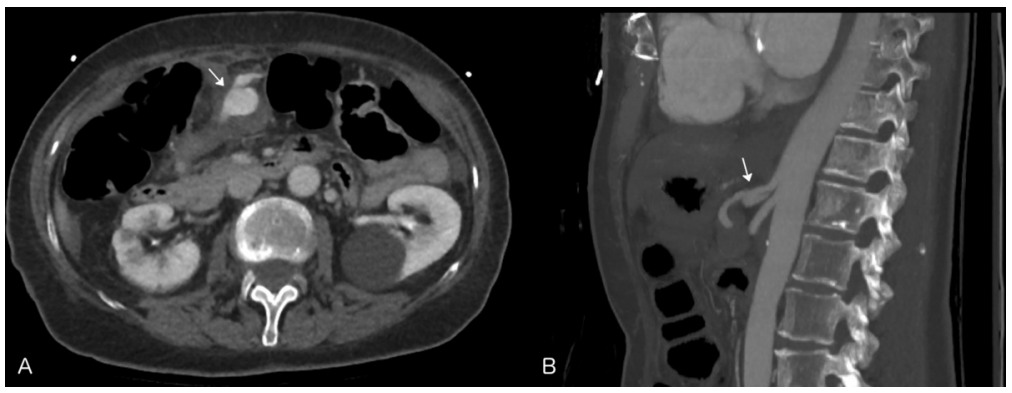

Figure 1 (A) Axial CT abdominal angiogram (delayed phase) pre-procedure: $20 \mathrm{~mm}$ pseudoaneurysm of the gastroduodenal artery (white arrow) with surrounding haematoma. (B) Sagittal CT abdominal angiogram (arterial phase) pre-procedure: fusiform aneurysm and dissection flap of the coelic axis (white arrow). 


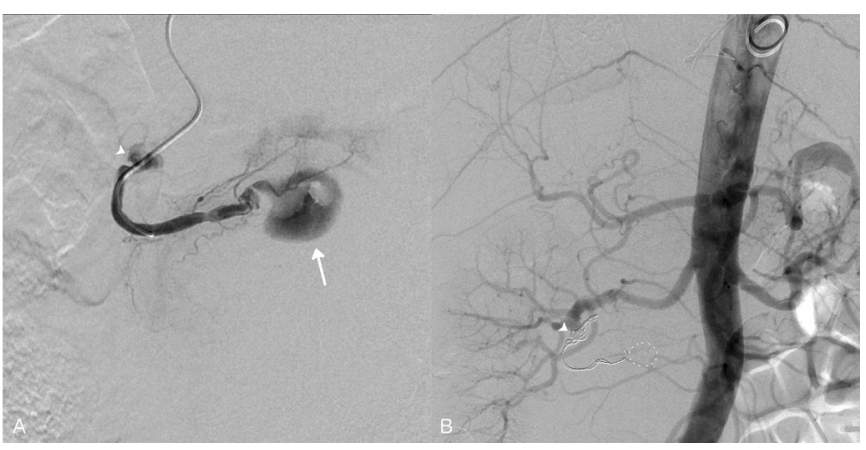

Figure 2 (A) Catheter angiogram of the gastroduodenal artery via the coeliac axis: proximal and distal pseudoaneurysms of the gastroduodenal artery (smaller proximal pseudoaneurysm marked by white arrowhead; larger distal pseudoaneurysm marked by white arrow). (B) Catheter angiogram post-coil embolisation: no flow into the gastroduodenal pseudoanerysms (site of smaller proximal pseudoaneurysm marked by white arrowhead; site of larger distal pseudoaneurysm outlined by dotted white oval).

\section{OUTCOME AND FOLLOW-UP}

The patient's systolic blood pressure was maintained at less than $120 \mathrm{~mm} \mathrm{Hg}$ throughout her 9-day admission, and her abdominal pain subsided following embolisation. At 12 months, the patient remained asymptomatic with a stable CT appearance of the vascular lesions.

\section{DISCUSSION}

Approximately 100 cases of SAM have been reported since it was first described by Slavin and Gonzalez Vitale in $1976 .{ }^{1}$ The differential diagnoses considered for this patient's presentation included infectious, inflammatory and non-infectious/inflammatory aetiologies. The patient had not been febrile, was not an intravenous drug user and had no clinical features of infection. Blood and stool cultures were negative, as were HIV, hepatitis $\mathrm{B}$ and $\mathrm{C}$, and syphilis serology. In addition, the patient did not show systemic features suggesting a vasculitic process (negative anti-neutrophil cytoplasmic antibody, antinuclear antibody, double-stranded DNA, rheumatoid factor and antiphospholipid panel). The patient also did not fit the American Rheumatology Association's criteria for polyarteritis nodosa. ${ }^{2}$

In considering non-inflammatory and non-infectious differentials, Ehlers Danlos, neurofibromatosis and fibromuscular dysplasia are not commonly seen in this age group. Physical and radiological features of Marfan's syndrome were absent, and the distribution of the lesions was against a diagnosis of atherosclerosis.

The radiological findings and the exclusion of other causes lead to the diagnosis of SAM. The presentation of SAM ranges from haemodynamic shock due to rupture of aneurysms, and can be an incidental finding in asymptomatic patients. ${ }^{13}$ SAM commonly involves the splanchnic (typically SMA and coeliac) arteries of middle-aged and elderly adults, and less frequently the intracranial, carotid, renal and iliac arteries. ${ }^{4}$ The non-inflammatory lytic degeneration of medial smooth muscle can result in stenosis, occlusion, dissection, aneurysm or pseudoaneurysm formation. ${ }^{24}$ SAM tends to have 'spatiotemporal characteristics', with numerous vessels affected at different time points. Several parts of one artery can also be affected, causing segmental and skip patterns, ${ }^{45}$ as seen in this patient.

Radiological evaluation with CT angiogram or conventional angiogram is essential. ${ }^{367}$ Multiple aneurysms can typically
Patient's perspective

Leaving Mildura in the middle of the night to be flown by the Royal Flying Doctor Service to Melbourne left me somewhat anxious, as staff in Mildura had been unable to tell me what the problem was; however, the paramedics tried to make me comfortable during the trip. On arriving at the Alfred Hospital, I was reassured by the professionalism of the doctors and staff.

The doctors and nursing staff kept me well informed of all the procedures and what they had found during subsequent scans. When the first procedure was not successful, the surgeon came up to the room and explained what they had done and what was happening. They were going to take some time to analyse the next step and had sought further advice elsewhere as this was a somewhat rare occurrence.

My family, I found out later, had also been kept up to date at all times. The second op which was two-thirds successful and involved coiling to two of the main aneurysms went without issues. At all times, the doctors and nursing staff made me feel very special and well informed. I might add that the follow-up since leaving the hospital to check on my well-being has been extremely well received, once again I thank you all for the wonderful care that I received at the Alfred.

\section{Learning points}

- Segmental arterial mediolysis (SAM) is a rare but important cause of spontaneous intra-abdominal haemorrhage where inflammatory disease and infection have been excluded.

- SAM should be considered when multiple vascular lesions are identified in medium-to-large vessels, especially when more than one vessel is involved.

- If SAM is suspected in a patient with intra-abdominal haemorrhage, a multidisciplinary approach involving interventional radiology, vascular surgery and general surgery is recommended.

show a 'string of beads' appearance. Another key radiological feature is the absence of inflammation. ${ }^{89} \mathrm{~A}$ histological diagnosis is usually difficult to obtain and, as studies have shown, is not a prerequisite for a diagnosis especially if the above radiological characteristics are present. ${ }^{10}$

No formal guidelines exist for the management of SAM. Asymptomatic patients are managed medically with antiplatelet therapy and strict blood pressure control. In the setting of acute bleeding, angiographic treatment is the mainstay and preferred over laparotomy. This usually involves coil embolisation and sometimes a covered stent. Emergency laparotomy is reserved for life-threatening haemorrhage in the unstable patient. Follow-up of these lesions depends on baseline findings. Aneurysms require surveillance and may need elective angiographic intervention (embolisation/stent). Some authors argue that embolisation of incidentally found SAM is indicated due to the $50 \%$ mortality rate associated with rupture in the acute phase ${ }^{1112}$; however, there are insufficient data to support this.

Contributors All persons who meet authorship criteria are listed as authors, and all authors certify (as per submitted document) that they have participated sufficiently in the work to take public responsibility for the content. MMB: involved in the concept and design of the case. Carried out literature search. Involved in drafting the article and revising it for intellectual content. Involved in final approval of the version to be published. Is in agreement to be accountable for the article 
and to ensure that all questions regarding the accuracy or integrity of the article are investigated and resolved. ML: involved in the concept and design of the case. Involved in revising the article for its intellectual content. Involved in final approval of the version to be published. Is in agreement to be accountable for the article and to ensure that all questions regarding the accuracy or integrity of the article are investigated and resolved. CM: involved in the concept and design of the case. Involved in revising the article for its intellectual content. Involved in final approval of the version to be published. Is in agreement to be accountable for the article and to ensure that all questions regarding the accuracy or integrity of the article are investigated and resolved. TJ: involved in the concept and design of the case. Involved in revising the article for its intellectual content. Involved in final approval of the version to be published. Is in agreement to be accountable for the article and to ensure that all questions regarding the accuracy or integrity of the article are investigated and resolved. JCL: involved in the concept and design of the case. Involved in revising the article for its intellectual content. Involved in final approval of the version to be published. Is in agreement to be accountable for the article and to ensure that all questions regarding the accuracy or integrity of the article are investigated and resolved.

Funding This research received no specific grant from any funding agency in the public, commercial or not-for-profit sectors.

Competing interests None declared.

Patient consent Obtained.

Provenance and peer review Not commissioned; externally peer reviewed.

(c) BMJ Publishing Group Ltd (unless otherwise stated in the text of the article) 2018. All rights reserved. No commercial use is permitted unless otherwise expressly granted.

\section{REFERENCES}

1 Slavin RE, Gonzalez-Vitale JC. Segmental mediolytic arteritis: a clinical pathologic study. Lab Invest 1976;35:23-9.

2 Lightfoot RW, Michel BA, Bloch DA, et al. The American College of Rheumatology 1990 criteria for the classification of polyarteritis nodosa. Arthritis Rheum 1990;33:1088-93.

3 Michael M, Widmer U, Wildermuth S, et al. Segmental arterial mediolysis: CTA findings at presentation and follow-up. AJR Am J Roentgenol 2006;187:1463-9.

4 Kuriyama A. Segmental arterial mediolysis. Am J Emerg Med 2017;35:518.e1-518.e2.

5 Slavin RE. Segmental arterial mediolysis: course, sequelae, prognosis, and pathologicradiologic correlation. Cardiovasc Pathol 2009;18:352-60.

6 Shimohira M, Ogino H, Sasaki S, et al. Transcatheter arterial embolization for segmental arterial mediolysis. J Endovasc Ther 2008;15:493-7.

7 Davran R, Cinar C, Parildar M, et al. Radiological findings and endovascular management of three cases with segmental arterial mediolysis. Cardiovasc Intervent Radiol 2010:33:601-6.

8 Garg N, Keller FS. Segmental arterial mediolysis, a vasculitis mimic: case report and review of literature. IJDI 2014;1:101-5.

9 Filippone EJ, Foy A, Galanis T, et al. Segmental arterial mediolysis: report of 2 cases and review of the literature. Am J Kidney Dis 2011;58:981-7.

10 Shenouda M, Riga C, Naji Y, et al. Segmental arterial mediolysis: a systematic review of 85 cases. Ann Vasc Surg 2014;28:269-77.

11 Soulen MC, Cohen DL, Itkin M, et al. Segmental arterial mediolysis: angioplasty of bilateral renal artery stenoses with 2-year imaging follow-up. J Vasc Interv Radiol 2004:15:763-7.

$12 \mathrm{Kim} \mathrm{HS}$, Min SI, Han A, et al. Longitudinal evaluation of segmental arterial mediolysis in splanchnic arteries: case series and systematic review. PLoS One 2016;11:e0161182.

Copyright 2018 BMJ Publishing Group. All rights reserved. For permission to reuse any of this content visit

http://group.bmj.com/group/rights-licensing/permissions.

BMJ Case Report Fellows may re-use this article for personal use and teaching without any further permission.

Become a Fellow of BMJ Case Reports today and you can:

- Submit as many cases as you like

- Enjoy fast sympathetic peer review and rapid publication of accepted articles

- Access all the published articles

Re-use any of the published material for personal use and teaching without further permission

For information on Institutional Fellowships contact consortiasales@bmjgroup.com

Visit casereports.bmi.com for more articles like this and to become a Fellow 